\title{
Conservación de la tortuga marina (Chelonia mydas) en Cozumel
}

DOI: $10.22403 /$ UQROOMX/TYP02/06

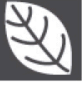

\section{RESUMEN}

Cada año en la isla de Cozumel se realiza el programa de protección de las tortugas marinas Caretta caretta y Chelonia mydas, especies que arriban a la costa oriental de la isla y cuya tasa de crecimiento está siendo afectada por la explotación ilegal de sus huevos. Pero en este artículo sólo se hablará de la especie Chelonia mydas; se expondrá parcialmente el ciclo de vida de la tortuga; su clasificación científica, distribución geográfica, su morfología tanto interna como externa. Asimismo, daremos información sobre la etapa de anidación de la tortuga blanca en la isla y se describirá en qué consiste el programa de Protección de la Tortuga Marina en Cozumel, Quintana Roo.

Palabras $\mid$ Cozumel, Chelonia mydas, anidación, eclosión, CLAVE protección de la tortuga marina, tortuga blanca.

* Universidad de Quintana Roo / Licenciatura en Manejo de Recursos Naturales (Especialidad en Costas) / georginamena@gmail.com 


\section{Introducción}

En la isla de Cozumel arriba la especie de tortuga marina Chelonia mydas (verde o blanca), actualmente amenazada y en peligro de extinción, debido principalmente a actividades antropogénicas como la intensa captura de tortugas adultas, en su mayoría hembras, para extraer de ellas alimentos (aceites y proteínas) y derivados (hueso, cuero y concha); el saqueo de huevos en las playas, motivado por mitos que les atribuyen poderes afrodisíacos; $y$ por los desarrollos turísticos costeros y pérdidas de hábitat de anidación (Frazier, 2000: 16-20). Esta notable disminución en las poblaciones ha ocasionado que las siete especies que anidan en México sean consideradas como especies en peligro de extinción de acuerdo con leyes nacionales e internacionales (NOM059-ECOL-200I y The World Conservation Union) (González-Baca, 2005: I).

En este artículo se describe parcialmente el ciclo de vida de la tortuga; su clasificación científica, distribución geográfica, su morfología tanto interna como externa, así también, daremos información sobre la etapa de anidación de la tortuga blanca en Cozumel (Semarnat, 2005). Describiremos también en qué consiste el programa de Protección de la Tortuga Marina en Cozumel, Quintana Roo.

\section{Ubicación taxonómica}

La tortuga Chelonia mydas se clasifica de la siguiente manera (Kart, 1999: 990-999):

$\begin{array}{ll}\text { Reino: } & \text { Animalia } \\ \text { Subreino: } & \text { Metazoa } \\ \text { Phylum: } & \text { Chordata } \\ \text { Subphylum: } & \text { Vertebrata } \\ \text { Superclase: } & \text { Tetrapoda } \\ \text { Clase: } & \text { Reptilia } \\ \text { Subclase: } & \text { Anapsida } \\ \text { Orden: } & \text { Testudinata }\end{array}$




$\begin{array}{ll}\text { Superfamilia: } & \text { Chelonioidea } \\ \text { Familia: } & \text { Cheloniidae (Chelonydae) } \\ \text { Género: } & \text { Chelonia } \\ \text { Especie: } & \text { Chelonia mydas }\end{array}$

\section{Morfología interna}

La morfología esquelética del cuerpo de las tortugas marinas tiene características especiales, la concha se forma con el crecimiento de placas óseas de origen dérmico que se fusionan dorsalmente a las costillas y a las vértebras, formando una dura bóveda; ésta continúa lateralmente en la parte ventral, con el plastrón, que se encuentra solamente articulado en los puentes, por medio de tejido conectivo cartilaginoso, que le permite cierta distensión en dirección vertical, facilitando los movimientos de la respiración (Pritchard y Mortimer, 2000: 23-4I).

Debido a que el cuerpo de la tortuga se encuentra encerrado en una caja, los huesos de las cinturas pectoral y pélvica se articulan y se reducen, así, las clavículas e interclavículas se fusionan al plastrón, de tal manera que adquieren una morfología muy especial que permite la retracción de los miembros dentro de esa caja. Las patas parecen remos, con los dedos unidos, muy largos y solamente con una o dos uñas reducidas. La cola es corta en las hembras; larga y prensil en los machos, es un apéndice cónico con una sola abertura cloacal en su base ventral. Las tortugas tienen respiración pulmonar, y los pulmones se encuentran adheridos a la superficie dorsal interna del carapacho. Debido a la ausencia de diafragma y de movimientos de la caja torácica, la inhalación y exhalación del aire se realiza mediante mecanismos tales que una inhalación completa es suficiente para que una tortuga marina permanezca en actividad bajo el agua durante 30 minutos sin mostrar efectos de anoxia. En estas inmersiones, y otras más prolongadas, intervienen mecanismos como la desaceleración del ritmo cardíaco (bradicardia), una especial habilidad del cerebro para seguir funcionando aun en concentraciones muy reducidas de oxígeno y una adaptación de las células sanguíneas (hematíes) para liberar el oxígeno hacia los tejidos en vez de absorberlo (Pritchard y Mortimer, 2000: 23-4I). 


\section{Morfología externa}

Su cabeza mide de 10 a 15 centímetros, con un par de escudos prefrontales. El caparazón tiene forma de corazón, puede alcanzar más de un metro de longitud, sin márgenes salientes, casi ovalado y angosto en la parte posterior, llega a medir entre 80 y $120 \mathrm{~cm}$ de largo, tiene cuatro pares de escudos laterales. El plastrón o peto con cuatro pares de escudos inframarginales, cinco centrales y II marginales; el peso corporal puede superar los $180 \mathrm{~kg}$. En las aletas tiene una sola uña (dos, en ocasiones). El color dorsal es verde oscuro o parduzco, con manchas radiadas amarillentas, verdes o negras y en la parte ventral una coloración blanca o amarillo pálido (fig. I). Se diferencia de otras tortugas marinas en que tiene un único par de placas en la parte superior de la cabeza (entre los ojos, un caparazón relativamente liso carente de resaltes) (Witzell, 1983: 78).
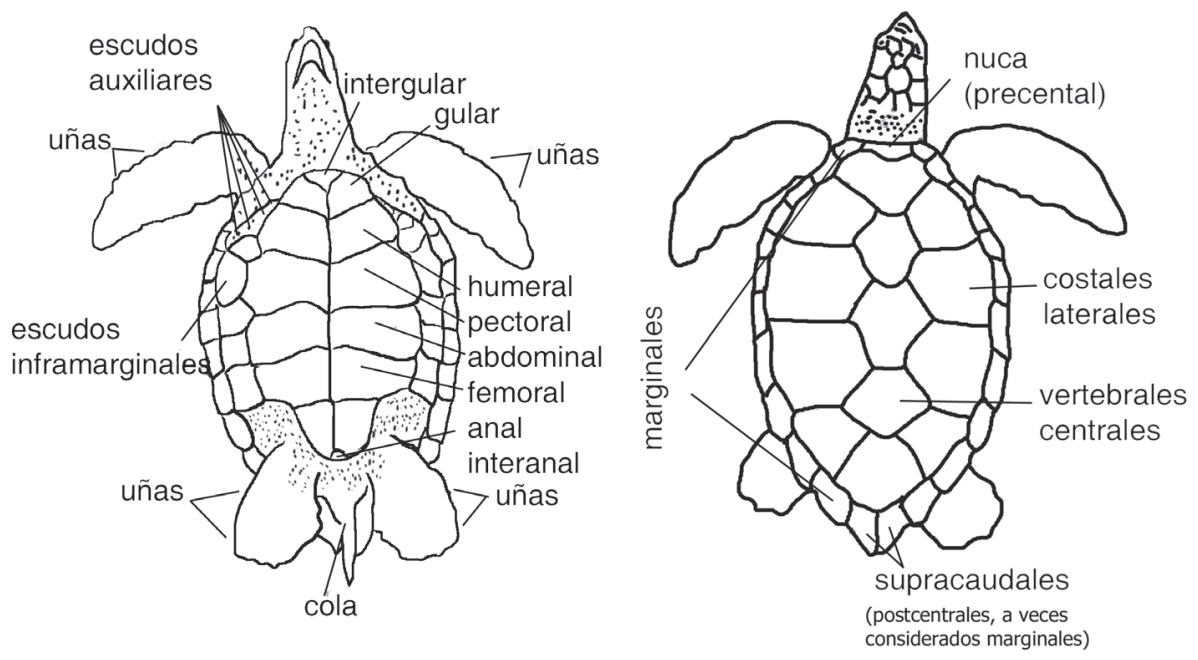

Figura I. Rasgos morfológicos externos de la tortuga Chelonia mydas

(Pritchard y Mortimer 2000:24) 


\section{Distribución mundial}

La principal colonia reproductiva de la especie a nivel global está localizada en la Isla de Raine, en Australia, donde hasta 80000 tortugas blancas anidan en un año. Chelonia mydas es la tortuga más abundante del mar Caribe, la zona de mayor nidificación es Playa de Tortuguero, en Costa Rica, donde se ha registrado un promedio de 23692 hembras nidificantes por año entre 197I y 198I. En Venezuela, anida a bajas densidades a lo largo de toda la costa y con mayor frecuencia en Falcón, Sucre, Nueva Esparta, archipiélago Los Roques y La Blanquilla. La mayor concentración se encuentra en Isla de Aves, donde cerca de 500 hembras desovan todos los años, siendo después de Tortuguero la segunda colonia reproductiva más grande en el mar Caribe (Pritchard y Mortimer, 2000: 23-4I).

Es una especie circuntropical que habita aguas que permanecen por encima de los $20^{\circ} \mathrm{C}$ durante todo el año. Aunque se encuentran en todo el planeta, sus poblaciones reproductivas están aisladas y desconectadas entre sí. Las hembras retornan a la playa donde nacieron para desovar, sin contacto alguno con otras colonias reproductivas. Esto ha llevado a sugerir que cada una de estas poblaciones debe ser considerada como una entidad genética única y aislada, lo que dificulta la aplicación del concepto tradicional de especie en este contexto (una entidad donde todos los individuos son capaces de cruzarse entre sí y producir progenie fértil) (Groombridge 1994: 8-19).

\section{Distribución en México}

En México, la anidación se distribuye desde Tamaulipas hasta Quintana Roo. En Tamaulipas, en Rancho Nuevo; entre Tampico alto y Tuxpan, Veracruz, en las playas: cayo Arenas, cayo Arcas y arrecife Triángulos; en Campeche, entre Isla Aguada y Sabancuy; en Yucatán, en Ría Lagartos y Coloradas, y en Quintana Roo, en Isla Mujeres, Isla Cozumel, X'cacel, Mahahual, Tulum y Chetumal (Semarnat, 2005) (véase fig. 2).

\section{Reproducción}

El macho nunca abandona el mar; la hembra sólo lo hace por la noche para poner sus huevos, de II0-II5 hasta 170 huevos. Anidan cada dos años o 


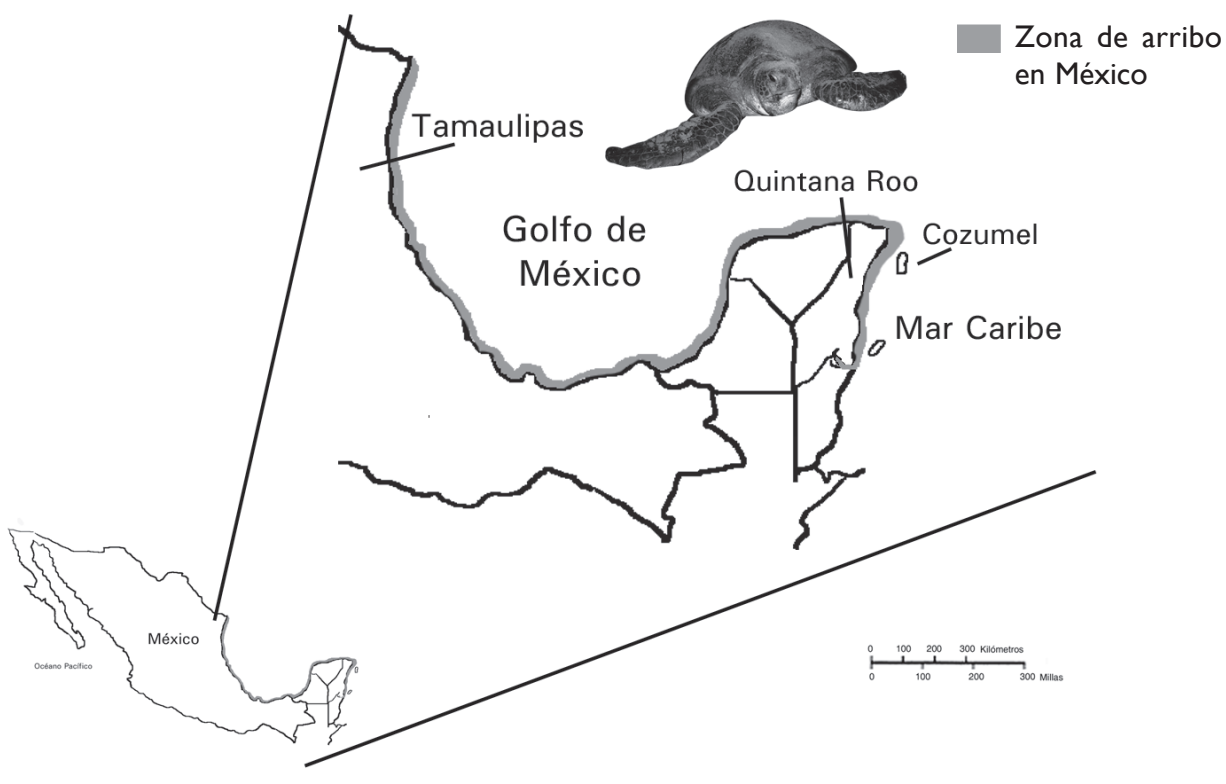

Figura 2. Zona de arribo de la tortuga marina en la República Mexicana.

más y ponen de tres a cinco veces por temporada, las cuales duran aproximadamente 60 días, la incubación se realiza más arriba de la marca de la marea alta. La temporada de anidación es entre mayo y octubre (época de máxima precipitación con influencia de ciclones) el nido llega a tener una profundidad de 60 a 70 centímetros. Los huevos son esféricos de 4.5 centímetros de diámetro (fig. 3).

Las crías salen de los huevos incubados por el calor del sol y no tardan en dirigirse hacia el mar (fig. 4). Debido a la sobre explotación, el número de tortugas verdes está disminuyendo rápidamente, por lo que es considerada una especie amenazada. Asimismo, la proporción de cada sexo en una nidada es determinada por la temperatura del medio. De todas las especies de tortugas marinas, se creen que la tortuga verde es la que tiene más longevidad, así que 
llega a la madurez sexual aún más tarde, entre 20 y 50 años. La tortuga verde es reconocida como una especie fiel a su lugar de anidación, lo cual significa que una tortuga regresará a la misma playa cada vez que esté lista para desovar (Pritchard y Mortimer, 2000: 23-4I).

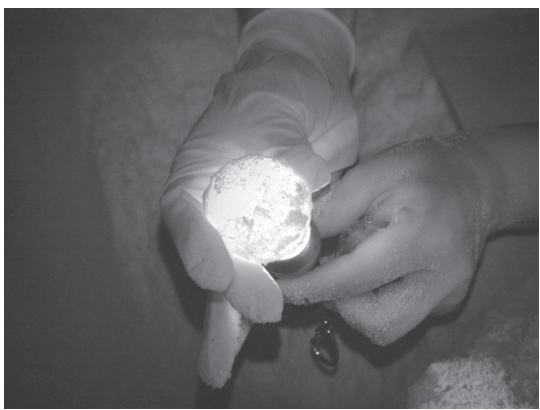

FIGURA 3. HUEVO CON 30 MINUTOS DE INCUBACIÓN

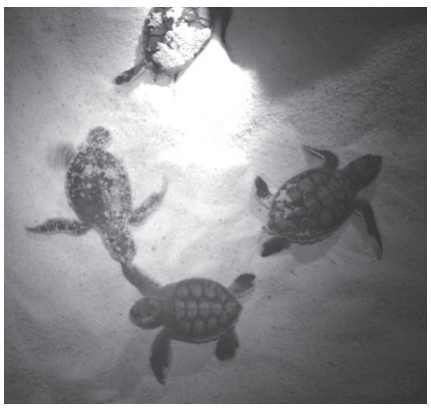

Figura 4. Crías de Chelonia mydas

\section{Identificación de rastros}

El tamaño, la huella y el tipo de rastro que deja cada una de las tortugas marinas al subir a la playa, es un indicio para saber de qué especie se trata. En el caso de la tortuga Chelonia mydas su tipo de huella es alternada, es decir, impulsan su cuerpo hacia delante con las aletas delanteras, mueven primero una y después la otra, formando una marca diagonal sobre el rastro dejado en la arena (fig. 5).

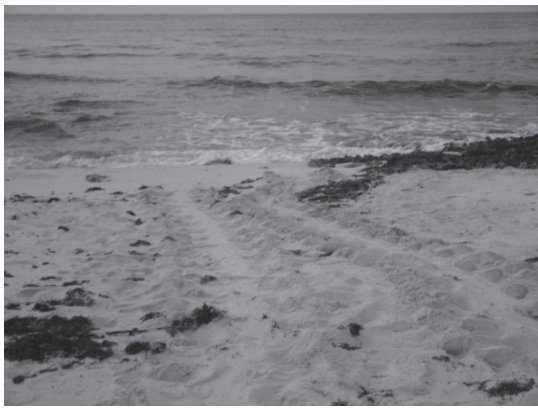

FIGURA 5. RASTRO DE TORTUGA BLANCA 


\section{Conservación}

En Cozumel, la organización de las labores de conservación ha sido ejemplar. Desde 1988 la conservación se ha realizado a través de un comité coordinado por el $\mathrm{H}$. Ayuntamiento. Este comité incluye grupos de diversos sectores (académico, empresarial, ciudadano e institucional) (González-Baca, 2005: I).

En Cozumel, la zona de mayor puesta de huevos de la tortuga blanca se localiza en la zona oriental de la isla; cada sector participa con una brigada, en la cual los estudiantes de la primera generación de la licenciatura en Manejo de los Recursos Naturales (donde participan los autores del presente escrito a través de la brigada de la Universidad de Quintana Roo) coadyuvan a la conservación de este quelonio, que esperamos siga regresando a las costas.

La brigada consta de 14 personas, que a su vez se dividen en dos grupos, que se concentran en la costa oriental de la isla todos los miércoles a partir de las nueve de la noche hasta las seis de la mañana del jueves. La temporada que aquí se describe comprendió del 14 de mayo hasta el 28 de octubre del 2005.

El trabajo de protección y conservación de la tortuga marina que realiza la brigada de la Universidad de Quintana Roo se describe a continuación.

El punto de partida para dirigirse a la costa oriental es en las instalaciones de la UQROO a las 9:00 p.m., donde a cada integrante se le asigna una tarea:

- Alumbrador. Su labor consiste en alumbrar la arena con una linterna cubierta con celofán rojo (para evitar el estrés que produce en las tortugas la luz blanca), a fin de detectar el rastro de la tortuga y ubicar los nidos.

- Buscador de nidos. Una vez localizados los nidos, se marca la ubicación exacta mediante la colocación de una varilla en forma de T. Luego se escarba el nido para cerciorarse de que se encuentren los huevos. Asimismo, por medio de los rastros en la arena, se identifica la especie de la tortuga puso los huevos.

- Anotador. Es la persona que lleva el control de los registros de los nidos localizados. En una libreta anota los siguientes datos: playa, fecha, especie, número de nido, hora, GPS, temperatura, distancias nido-costa y nido-carretera. 
- Medidores. Luego de localizar los huevos del nido, se procede a medir la distancia de la orilla del mar al nido y a la carretera y/o duna.

- Elaborador de marcas. Cuando se tiene la certeza de la ubicación de los huevos del nido se elaboran marcas, consistentes en cintas en las que se escribe la fecha de registro, la especie, el número consecutivo del nido y nombre de la brigada. Las marcas se entierran en el nido para su posterior ubicación (después de que eclosionen los huevos), y se colocan estacas en puntos estratégicos.

- Borradores. Para evitar confusiones en la localización de los nidos se borran los rastros que las tortugas dejan en la arena al salir del mar.

Cuando se llega a la costa oriental se hace el recorrido por carretera (empezando en la playa Mezcalitos y en dirección al sur hasta la playa Bosh) alumbrando desde el transporte la zona de arena para detectar los nidos. Una vez detectados se deja el transporte para ubicarlos exactamente.

Cuando se localizan los huevos, los brigadistas realizan las tareas antes mencionadas en un tiempo no mayor a 10 minutos por nido; hasta cubrir el tiempo de nueve horas.

Después de que se registran los nidos de tortuga, se calcula su fecha de eclosión para regresar en el momento preciso y determinar el nivel de natalidad y mortandad por nido, para lo cual se realiza un conteo de cascarones rotos (huevos eclosionados o tortugas vivas), tortugas muertas, embriones muertos y huevos sin desarrollo aparente.

Con este trabajo pretendemos contribuir al estudio, manejo y conservación de las especies que arriban a la isla de Cozumel, Quintana Roo.Asimismo, las brigadas que participamos en la temporada 2005 queremos mejorar las técnicas de localización de los nidos para su aplicación en temporadas futuras.

\section{Agradecimientos}

Los autores agradecen al Dr. Luis M. Mejía-Ortiz por sus correcciones al presente escrito y a la M. en C. Marilú López-Mejía por la fotografía de las crías de tortuga, así como al área de Ciencias del Departamento de Ciencias y Humanidades de la División de Desarrollo Sustentable de la UQROo-Cozumel, por su apoyo financiero a la brigada de la UQROO-Cozumel, en el programa de Protección a la Tortuga Marina coordinado por el H.Ayuntamiento de la Isla de Cozumel. 


\section{FUENTES CONSULTADAS}

Frazier, John G. (2000). “Conservación basada en la comunidad”, en K. L. Eckert et al. (eds.), Técnicas de investigación y manejo para la conservación de las tortugas marinas. Washington, D.C.: Grupo Especialista en Tortugas Marinas UICN/CSE, 16-20.

González-Baca, C. (2005). Reporte del comité de protección a la tortuga marina, 2005. México: Campamento Tortuguero y Centro de Interpretación Ambiental. Cozumel, p. 4.

Groombridge, B. (1994). Biodiversity Data Sourcebook. Cambridge, Inglaterra: World Conservation Press, 8-19.

Karl, S.A. y B.W. Bowen. (1999). “Evolutionarily significant units versus geopolitical taxonomy: molecular systematics of an endangered sea turtle (genus Chelonia)" Conservation Biology, 13, (5), 990-999.

Pritchard, P. C. H. y J. A. Mortimer (2000). “Taxonomía, morfología externa e identificación de las especies”, en K. L. Eckert et al. (eds.), Técnicas de investigación y manejo para la conservación de las tortugas marinas. Washington, D. C.: Grupo Especialista en Tortugas Marinas UICN/CSE, 23-4I.

Semarnat (2005). Secretaría de Medio Ambiente Recursos Naturales (en línea). Disponible en: http://www.semarnat.gob.mx/especies/tortuga/ verde.shtml [fecha de consulta: 5 de junio de 2005]. 